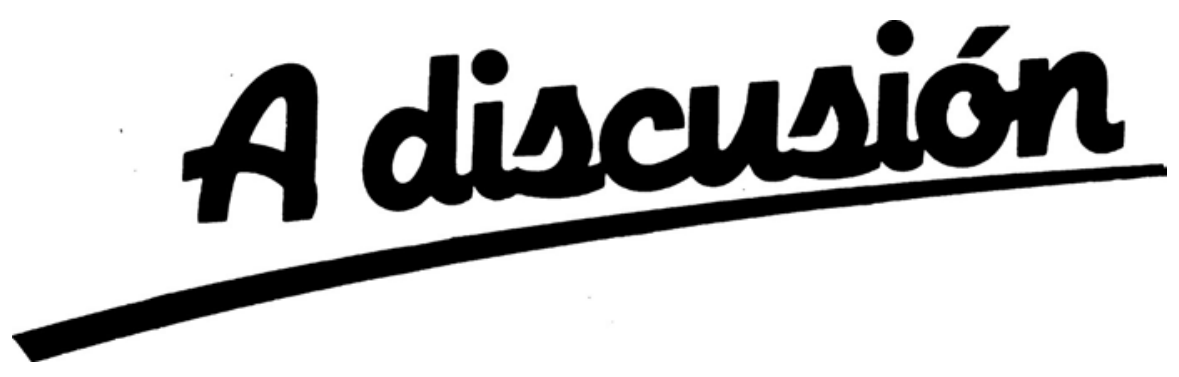

\title{
THE TALMUD RULE AND THE SECUREMENT OF AGENTS' AWARDS*
}

\section{Juan D. Moreno-Ternero and Antonio Villar**}

WP-AD 2003-05

Correspondence to: Juan D. Moreno-Ternero. Universidad de Alicante. Departamento de Fundamentos del Análisis Económico. Campus San Vicente del Raspeig, s/n. 03080 Alicante (Spain). Phone: (+) $34 \quad 96 \quad 590 \quad 36 \quad 14$ / Fax: (+) $34 \quad 96 \quad 590 \quad 38 \quad 98$ / E-mail: juande@merlin.fae.ua.es.

Editor: Instituto Valenciano de Investigaciones Económicas, S.A.

Primera Edición Febrero 2003

Depósito Legal: V-896-2003

IVIE working papers offer in advance the results of economic research under way in order to encourage a discussion process before sending them to scientific journals for their final publication.

\footnotetext{
* This work was done while the second author was visiting the European University Institute, thanks to a Salvador de Madariaga grant from the Ministerio de Educación, Cultura y Deporte. We thank Carmen Herrero and Dunia López-Pintado for helpful comments and suggestions. Financial support from the Ministerio de Ciencia y Tecnología, under project BEC2001-0535, and from the Generalitat Valenciana under project GV01-371, is gratefully acknowledged.
}

** J.D. Moreno-Ternero: University of Alicante. A. Villar: University of Alicante and Ivie. 


\title{
THE TALMUD RULE AND THE SECUREMENT OF AGENTS' AWARDS
}

\author{
Juan D. Moreno-Ternero and Antonio Villar
}

\begin{abstract}
This paper provides a new characterization of the Talmud rule by means of a new property, called securement. This property says that any agent holding a feasible claim will get at least one $n$ ht of her claim, where $n$ is the number of agents involved. We show that securement together with a weak version of path independence and the standard properties of self-duality and consistency characterize the Talmud rule.
\end{abstract}

Keywords: bankruptcy problems, Talmud rule, characterization results.

J.E.L. Classification: D63 


\section{Introduction}

A bankruptcy problem describes a situation in which an arbitrator has to allocate a given amount of a perfectly divisible commodity among a group of agents, when the available amount is not enough to satisfy all their claims. Some standard examples of this type of problem are: the bankruptcy of a firm, the execution of a will with insufficient assets, the collection of a given amount of taxes, the allocation of equities in privatized firms, the distribution of commodities in a fixed-price setting, and sharing the cost of an indivisible public facility. A solution to a bankruptcy problem is a procedure or "rule" that satisfies some desirable properties and determines, for each specific problem, an allocation in which no agent gets more than she claims nor less than zero. The reader is referred to the works of Young (1994, ch. 4), Herrero \& Villar (2001), Moulin (2002) and Thomson (2002) for a review of this literature.

Different rules are usually associated with alternative sets of properties that represent ethical and operational principles. The object of the axiomatic approach to bankruptcy problems is, precisely, to identify each rule with a well defined set of properties. This helps understanding the nature of the different solutions and their applicability. It is therefore interesting to have alternative characterizations of the same rule, as this permits one to have different insights on the principles underlying the rule and on the type of problems for which it might be suitable.

This paper follows the axiomatic approach focusing on one specific solution concept: the Talmud rule. This rule was proposed by Aumann \& Maschler (1985) as the consistent extension of the so called "contested garment rule", a solution concept defined for two-person bankruptcy problems. The contested garment rule concedes to each agent her minimal right (to be understood as what is left, if anything, when the other agent gets her claim fully honored) and then divides equally the reminder. The reader is referred to Dagan (1996) for a detailed study of this rule.

In this paper we introduce a new property, called securement, that provides a protective criterion which ensures each agent a minimal share of her individual claim, no matter what the other claims are. More precisely, securement says that any agent holding a feasible claim (a claim not larger than the estate) will get at least one $n$th of her claim, where $n$ is the number of agents involved.

We also provide a weakening of Moulin's (1987) notion of path independence, called restricted path independence, that refers to the behavior of the rule when the estate turns out to be smaller than expected, but only applies when no claim is feasible. It requires the solution of the actual problem to 
be the same as that which takes the (unfeasible) allocation corresponding to the division of the estimated estate as the new claims point.

We show that the only consistent rule that satisfies securement, restricted path independence and self-duality is the Talmud rule. Moreover, all these properties are independent.

The rest of the paper is organized as follows. The model and the preliminary definitions are introduced in Section 2. The characterization result is presented in Section 3. Finally, Section 4 concludes providing further insights on nature of the characterization result.

\section{$2 \quad$ Model and definitions}

Let $\mathbb{N}$ represent the set of all potential agents (a set with an infinite number of members) and let $\mathcal{N}$ be the family of all finite subsets of $\mathbb{N}$. An element $N \in \mathcal{N}$ describes a finite set of agents $N=\{1,2, \ldots, n\}$, where we take $|N|=n$. A bankruptcy problem [O'Neill (1982)] is a triple $(N, E, c)$, where $N$ is the set of agents, $E \in \mathbb{R}_{+}$represents the estate (the amount to be divided), and $c \in \mathbb{R}_{+}^{n}$ is a vector of claims whose $i$ th component is $c_{i}$, with $\sum_{i \in N} c_{i}>E>0$. The family of all those bankruptcy problems is $\mathbb{B}$. To simplify notation we write, for any given problem $(N, E, c) \in \mathbb{B}$, $C=\sum_{i \in N} c_{i}$ and $L=C-E$. We assume, without loss of generality, that $c_{1} \leq c_{2} \leq \ldots \leq c_{n}$. We say that the $i$ th agent's claim is feasible if $c_{i} \leq E$.

A rule is a mapping $R$ that associates with every $(N, E, c) \in \mathbb{B}$ a unique point $R(N, E, c) \in \mathbb{R}^{n}$ such that $0 \leq R(N, E, c) \leq c$ and $\sum_{i \in N} R_{i}(N, E, c)=$ $E$. The point $R(N, E, c)$ represents a desirable way of dividing $E$ among the agents in $N$ so that each agent receives an award that is non-negative and bounded above by her claim, and the entire estate is allocated.

We focus here on a specific rule, the Talmud rule [Aumann \& Maschler (1985)], which is defined as follows:

Definition 1 The Talmud $(\mathbf{T})$ is the rule that, for all $(N, E, c) \in \mathbb{B}$, and all $i \in N$, yields:

$$
T_{i}(N, E, c)= \begin{cases}\min \left\{\frac{1}{2} c_{i}, \lambda\right\} & \text { if } E \leq \frac{1}{2} C \\ \max \left\{\frac{1}{2} c_{i}, c_{i}-\mu\right\} & \text { if } E \geq \frac{1}{2} C\end{cases}
$$

where $\lambda$ and $\mu$ are chosen so that $\sum_{i \in N} T_{i}(N, E, c)=E$.

Besides being the consistent extension of the contested garment rule, the Talmud rule is a sensible allocation procedure on its own which makes the allocation formula to depend on the relative size of the estate and the claims. 
More specifically, the Talmud rule establishes that nobody will get more than half her claim if the estate is below half the aggregate claim, and nobody will lose more than half her claim if the estate is above half the aggregate claim.

Let us now consider three standard properties that are satisfied by this rule. The first one, self-duality, says that gains and losses are treated on an equal foot. The second property, bilateral consistency, stipulates that if we apply a rule $R$ to a given problem $(N, E, c)$ or we do so to any of the associated reduced two-person problems, all incumbent agents get the same outcome. Bilateral consistency is a procedural requirement that summarizes the following principle: what is good for the large group is also good for the smallest one. One can also consider the converse of this property. "Converse consistency" establishes that the way of solving a two-person problem can be extended to any number of them. Hence, what is good for the smallest possible group is good for larger ones. That is our third property. Formally:

Definition 2 A rule $R$ satisfies self-duality if, for all $(N, E, c) \in \mathbb{B}, F(N, E, c)=$ $c-F(N, C-E, c)$.

Definition 3 A rule $R$ satisfies bilateral consistency if, for all $(N, E, c) \in$ $\mathbb{B}$, all $S \subset N$ with $|S|=2$, and all $i \in S$, we have: $R_{i}(N, E, c)=R_{i}\left(S, E_{S}, c_{S}\right)$, where $E_{S}=\sum_{i \in S} R_{i}(N, E, c)$ and $c_{S}=\left(c_{i}\right)_{i \in S}$.

Definition $4 A$ rule $R$ is converse consistent if, for each $(N, E, c) \in \mathbb{B}$ with $|N| \geq 3$ and each $x \in \mathbb{R}_{+}^{n}$ with $\sum_{i \in N} x_{i}=E$, if for each $S \subset N$ with $|S|=2, x_{S}=R\left(S, E-\sum_{i \in N \backslash S} x_{i}, c_{S}\right)$, then $x=R(N, E, c)$.

We now introduce two new properties, securement and restricted path independence. The first says that each agent holding a feasible claim should receive at least one $n$th of her claim. This property is reminiscent of the extreme protection criteria of exemption [Herrero \& Villar (2001)], that leads to the constrained equal awards rule. The second is a weakening of the notion of path independence [Moulin (1987)], and refers to the behavior of the rule when the estate turns out to be smaller than expected and no claim is feasible. Formally:

Definition 5 A rule satisfies securement if, for all $(N, E, c) \in \mathbb{B}, c_{i} \leq E$ implies $R_{i}(N, E, c) \geq \frac{1}{n} c_{i}$.

Securement is a property that guarantees a minimal share to those agents holding feasible claims. Note that this share only depends on the $i$ th agent's claim and the number of creditors involved. Namely, if a rule $R$ satisfies this property, agent $i$ will know the smallest amount she can get even without having any information on the other agents' claims. 
Definition 6 A rule $R$ satisfies restricted path independence if for all $(N, E, c) \in \mathbb{B}$, all $E^{\prime}$ such that $E \leq E^{\prime} \leq c_{1}$, we have

$$
R(N, E, c)=R\left[N, E, R\left(N, E^{\prime}, c\right)\right]
$$

Restricted path independence can be interpreted as follows. Suppose that the arbitrator makes an estimate $E^{\prime}$ of the estate knowing that there is no feasible claim in the problem, and makes a proposal $R\left(N, E^{\prime}, c\right)$. Then it turns out that this estimate is too optimistic and the actual estate is $E<$ $E^{\prime}$. Restricted path independence requires that solving the actual problem $(N, E, c)$ produces the same outcome as that in which one takes the initial (unfeasible) allocation of $E^{\prime}$ as the new claims point and then distributes the actual estate $E$.

\section{The characterization result}

The following preliminary result, which is interesting on its own, shows that in the case of two-agent problems equal treatment of equals is inferred from self-duality, securement and restricted path independence. ${ }^{1}$

Lemma 1 For two-agent bankruptcy problems, self-duality, securement and restricted path independence imply equal treatment of equals.

\section{Proof.}

Consider $\left(N, E,\left(c_{1}, c_{2}\right)\right)$ a two-agent bankruptcy problem, whose claims are equal, i.e., $c_{1}=c_{2}=z$. Let $R$ be a rule satisfying self-duality, securement and restricted path independence. Let us see that $R_{1}(N, E,(z, z))=$ $R_{2}(N, E,(z, z))$. Several cases are to be considered.

Case 1.- $E=z$. In this case, since $R$ satisfies securement,

$$
R_{i}(N, E,(z, z)) \geq \frac{z}{2}=\frac{E}{2}, \text { for } i=1,2 .
$$

Now, $R_{1}(N, E,(z, z))+R_{2}(N, E,(z, z))=E$ implies that $R_{i}(N, E,(z, z))=$ $\frac{E}{2}$, for $i=1,2$.

Case 2.- $E<z$. Let us denote by $n_{1}$ the minimum positive integer for which $\left(\frac{1}{2}\right)^{n_{1}} \cdot z<E$. Now, let us denote by $m_{1}$ the minimum positive integer

\footnotetext{
${ }^{1}$ Let us recall that a rule $R$ satisfies equal treatment of equals if, for all $(N, E, c) \in \mathbb{B}$, $c_{i}=c_{j}$ implies $R_{i}(N, E, c)=R_{j}(N, E, c)$.
} 
for which $E<\left(\frac{1}{2}\right)^{n_{1}} \cdot z \cdot \sum_{j=0}^{m_{1}}\left(\frac{1}{2}\right)^{j}$. Since $R$ satisfies self-duality and restricted path independence,

$$
R(N, E,(z, z))=\left(a_{1}, a_{1}\right)+R\left(N, E_{1},\left(d_{1}, d_{1}\right)\right),
$$

where $a_{1}=\left(\frac{1}{2}\right)^{n_{1}+1} \cdot z \cdot \sum_{j=0}^{m_{1}-1}\left(\frac{1}{2}\right)^{j}, E_{1}=E-\left(\frac{1}{2}\right)^{n_{1}} \cdot z \cdot \sum_{j=0}^{m_{1}-1}\left(\frac{1}{2}\right)^{j}$, and $d_{1}=\left(\frac{1}{2}\right)^{n_{1}+m_{1}} \cdot z$. Since $1 \leq \min \left\{n_{1}, m_{1}\right\}$, then $d_{1} \leq \frac{1}{4} z$. Notice that now $E_{1}<d_{1}$ and we can apply the same argument. After a finite number of iterations, say $K$, we would have

$$
R(N, E,(z, z))=\left(\sum_{k=1}^{K} a_{k}, \sum_{k=1}^{K} a_{k}\right)+R\left(N, E_{K},\left(d_{K}, d_{K}\right)\right),
$$

where $d_{K} \leq\left(\frac{1}{4}\right)^{K} \cdot z$. As a result, $R_{i}\left(N, E_{K},\left(d_{K}, d_{K}\right)\right) \leq\left(\frac{1}{4}\right)^{K} \cdot z$, for $i=1,2$. If we take limits, when $K \rightarrow \infty$, then

$$
R(N, E,(z, z))=\left(\sum_{k=1}^{\infty} a_{k}, \sum_{k=1}^{\infty} a_{k}\right),
$$

which implies $R_{1}(N, E,(z, z))=R_{2}(N, E,(z, z))$.

Case 3.- $E>z$. Let us denote by $r_{1}$ the minimum positive integer for which $z \cdot \sum_{j=0}^{r_{1}+1}\left(\frac{1}{2}\right)^{j}>E$. Now, let us denote by $s_{1}$ the minimum positive integer for which $E<\left(\left(\frac{1}{2}\right)^{s_{1}+r_{1}+1}+\sum_{j=0}^{r_{1}}\left(\frac{1}{2}\right)^{j}\right) \cdot z$. Since $R$ satisfies selfduality and restricted path independence,

$$
R(N, E,(z, z))=\left(b_{1}, b_{1}\right)+R\left(N, E_{1},\left(e_{1}, e_{1}\right)\right),
$$

where $b_{1}=\frac{1}{2} \cdot z \cdot \sum_{j=0}^{r_{1}}\left(\frac{1}{2}\right)^{j}, E_{1}=E-z \cdot \sum_{j=0}^{r_{1}}\left(\frac{1}{2}\right)^{j}$, and $e_{1}=\left(\frac{1}{2}\right)^{s_{1}+r_{1}+1} \cdot z$. Since $1 \leq \min \left\{r_{1}, s_{1}\right\}$, then $e_{1} \leq \frac{1}{4} z$. Notice that now $E_{1}>e_{1}$ and we can apply the same argument. After a finite number of iterations, say $K$, we would have

$$
R(N, E,(z, z))=\left(\sum_{k=1}^{K} b_{k}, \sum_{k=1}^{K} b_{k}\right)+R\left(N, E_{K},\left(e_{k}, e_{k}\right)\right),
$$

where $e_{k} \leq\left(\frac{1}{4}\right)^{K} \cdot z$. As a result, $R_{i}\left(N, E_{K},\left(e_{k}, e_{k}\right)\right) \leq\left(\frac{1}{4}\right)^{K} \cdot z$, for $i=1,2$. If we take limits, when $K \rightarrow \infty$, then

$$
R(N, E,(z, z))=\left(\sum_{k=1}^{\infty} b_{k}, \sum_{k=1}^{\infty} b_{k}\right),
$$

which implies $R_{1}(N, E,(z, z))=R_{2}(N, E,(z, z))$.

The main result of the paper is the following: 
Theorem 1 A bankruptcy rule $R$ satisfies bilateral consistency, self-duality, securement and restricted path independence if and only if it is the Talmud rule.

\section{Proof.}

It is well known that the Talmud rule satisfies bilateral consistency and self-duality [e.g. Herrero \& Villar (2001)]. Let us first see that the Talmud rule satisfies securement and restricted path independence, and then prove the converse implication.

(i) T satisfies securement.

Let $i \in N$ be such that $c_{i} \leq E$. As the property trivially holds when $E \geq \frac{1}{2} C$, let us assume that $E \leq \frac{1}{2} C$. In this case, $T_{i}(N, E, c)=\min \left\{\frac{1}{2} c_{i}, \lambda\right\}$. Now suppose, for the sake of contradiction, that $T_{i}(N, E, c)=\lambda<\frac{1}{n} c_{i} \leq \frac{1}{2} c_{i}$. Thus, $\lambda<\frac{E}{n}$. Now, for all $k \geq i$ we have $c_{k} \geq c_{i}$, which implies that $\frac{1}{2} c_{k}>\lambda$ and therefore $T_{k}(N, E, c)=\lambda<\frac{E}{n}$. As a consequence,

$E=\sum_{k \in N} T_{k}(N, E, c)=\sum_{k=1}^{i-1} T_{k}(N, E, c)+\sum_{k=i}^{n} \lambda<\sum_{k=1}^{i-1} T_{k}(N, E, c)+(n-i+1) \frac{E}{n}$.

Thus, $\sum_{k=1}^{i-1} T_{k}(N, E, c)>\frac{i-1}{n} E$. Since $T_{k}(N, E, c) \geq 0$, for all $k \in N$, then there exists some $k_{0} \leq i-1$ such that $T_{k_{0}}(N, E, c)>\frac{E}{n}>\lambda=T_{n}(N, E, c)$, which is a contradiction, since $T$ is an order preserving rule [c.f. Aumann \& Maschler (1985)].

(ii) $T$ satisfies restricted path independence.

Let $(N, E, c),\left(N, E^{\prime}, c\right) \in \mathbb{B}$ be such that $c_{1} \geq E^{\prime} \geq E$. Then, $E \leq E^{\prime} \leq$ $\frac{1}{2} C$, which implies $T_{i}(N, E, c)=\min \left\{\frac{1}{2} c_{i}, \lambda\right\}$ and $T_{i}\left(N, E^{\prime}, c\right)=\min \left\{\frac{1}{2} c_{i}, \lambda^{\prime}\right\}$ for all $i \in N$, where $\lambda$ and $\lambda^{\prime}$ are chosen so that $\sum_{i \in N} T_{i}(N, E, c)=E$, and $\sum_{i \in N} T_{i}\left(N, E^{\prime}, c\right)=E^{\prime}$.

Now, it is straightforward to show that

$$
T_{1}(N, E, c)= \begin{cases}\frac{E}{n} & \text { if } E \leq \frac{n}{2} c_{1} \\ \frac{1}{2} c_{1} & \text { if } \frac{n}{2} c_{1} \leq E \leq C-\frac{n}{2} c_{1} \\ c_{1}-\frac{C-E}{n} & \text { if } C-\frac{n}{2} c_{1} \leq E\end{cases}
$$

Since $c_{1} \geq E$, and according to (1), $T_{1}(N, E, c)=\frac{E}{n}$. Thus, $\frac{E}{n}=\lambda<\frac{1}{2} c_{1}$ and therefore $T_{i}(N, E, c)=\frac{E}{n}$, for all $i \in N$. Analogously, $T_{i}\left(N, E^{\prime}, c\right)=\frac{E^{\prime}}{n}$, for all $i \in N$. Consequently, since the Talmud rule satisfies equal treatment of equals, $T_{i}\left(N, E, T\left(N, E^{\prime}, c\right)\right)=\frac{E}{n}$, for all $i \in N$. Thus, $T_{i}\left(N, E, T\left(N, E^{\prime}, c\right)\right)=$ $T_{i}(N, E, c)$ for all $i \in N$, as desired. 
(iii) Bilateral consistency, self-duality, securement and restricted path independence imply the Talmud rule.

First note that the Talmud rule is converse consistent. Therefore, it is enough to prove the result for the two-agent case and then invoke the 'Elevator Lemma' [c.f. Thomson (1996)] to prove the result. ${ }^{2}$

Let $R$ be a rule that satisfies all those properties. Lemma 1 ensures that $R$ also satisfies equal treatment of equals. Without loss of generality let $(N, E, c)$ be a bankruptcy problem with $N=\{1,2\}$, and $c_{1} \leq c_{2}$. In these circumstances $T$ can be expressed as:

$$
T(N, E, c)=\left\{\begin{array}{ll}
\left(\frac{E}{2}, \frac{E}{2}\right) & \text { if } E \leq c_{1} \\
\left.\frac{c_{1}}{2}, E-\frac{c_{1}}{2}\right) & \text { if } c_{1} \leq E \leq c_{2} \\
\left.c_{1}-\frac{C-E}{2}, c_{2}-\frac{C-E}{2}\right) & \text { if } c_{2} \leq E
\end{array} .\right.
$$

There are several cases to be discussed.

Case 1.- $c_{1} \leq E \leq c_{2}$.

Since $c_{1} \leq E$ and $R$ satisfies securement then $R_{1}(N, E, c) \geq \frac{c_{1}}{2}$. Furthermore, $E \leq c_{2}$ is equivalent to saying that $c_{1} \leq L=C-E$. Thus, securement also implies that $R_{1}(N, L, c) \geq \frac{c_{1}}{2}$. Now, since $R$ is a self dual rule, $R_{1}(N, E, c)=c_{1}-R_{1}(N, L, c) \leq \frac{c_{1}}{2}$. As a result, $R(N, E, c)=\left(\frac{c_{1}}{2}, E-\frac{c_{1}}{2}\right)=$ $T(N, E, c)$.

Case 2.- $E<c_{1}$.

Thanks to Case $1, c^{\prime}=R\left(N, c_{1}, c\right)=\left(\frac{c_{1}}{2}, \frac{c_{1}}{2}\right)$. Now, since $R$ satisfies equal treatment of equals, $R\left(N, E, c^{\prime}\right)=\left(\frac{E}{2}, \frac{E}{2}\right)=T(N, E, c)$. Finally, $R\left(N, E, c^{\prime}\right)=R(N, E, c)$, thanks to restricted path independence.

Case 3.- $c_{2}<E$.

This case is equivalent to $c_{1}>L$. Thanks to Case $2, R(N, L, c)=$ $\left(\frac{L}{2}, \frac{L}{2}\right)$. By self-duality, $R(N, L, c)=c-R(N, E, c)$. Thus, $R(N, E, c)=$ $\left(c_{1}-\frac{L}{2}, c_{2}-\frac{L}{2}\right)=T(N, E, c)$.

\section{Discussion}

Three different questions are analyzed in this section, in order to dwell on the nature of the result in Theorem 1 . The first refers to the specificity of the securement property, which is the main ingredient of the characterization result (one may wonder whether this is an ad hoc property that only the Talmud rule satisfies, among the rules which are standard in the literature). The second regards the logical independence of the properties in the Theorem

\footnotetext{
${ }^{2}$ The 'Elevator Lemma' says that if a bilaterally consistent rule coincides with a conversely consistent rule in the two-agent case, coincidence holds in general.
} 
(the tightness of the characterization). Finally, the third deals with the comparison with other characterization results in the literature (namely: is it really a different characterization?).

To answer all these questions we analyze the performance of three well known rules with respect to the four properties in Theorem 1. These rules are the proportional rule, the constrained equal awards rule and the adjusted proportional rule. ${ }^{3}$ They are defined as follows:

Definition 7 The proportional rule $(P)$ yields for all $(N, E, c) \in \mathbb{B}$, and all $i \in N$ :

$$
P_{i}(N, E, c)=\frac{E}{C} \cdot c_{i} .
$$

Definition 8 The constrained equal awards rule $(A)$ yields for all $(N, E, c) \in$ $\mathbb{B}$, and all $i \in N$ :

$$
A_{i}(N, E, c)=\min \left\{c_{i}, \lambda\right\},
$$

where $\lambda$ is chosen so that $\sum_{i \in N} A_{i}(N, E, c)=E$.

In order to define properly the adjusted proportional rule, we need an additional piece of notation. Given a bankruptcy problem $(N, E, c) \in \mathbb{B}$, we define its vector of minimal rights as $m(N, E, c)=\left(m_{i}(N, E, c)\right)_{i \in N}$, and its vector of truncated claims as $t(N, E, c)=\left(t_{i}(N, E, c)\right)_{i \in N}$, where

$$
\begin{aligned}
m_{i}(N, E, c) & =\max \left\{0, E-\sum_{j \in N-\{i\}} c_{j}\right\} \text { for all } i \in N, \text { and } \\
t_{i}(N, E, c) & =\min \left\{c_{i}, E\right\} \text { for all } i \in N .
\end{aligned}
$$

Definition 9 The adjusted proportional rule $(A P)$ yields, for all $(N, E, c) \in$ $\mathbb{B}$, all $i \in N$ :

$$
A P_{i}(N, E, c)=m_{i}(N, E, c)+P\left(t\left(N, E-\sum_{i \in N} m_{i}(N, E, c), c-m(N, E, c)\right)\right),
$$

where $P$ is the proportional rule.

In words: the $A P$ rule assigns first to each agent her minimal right. Second, each agent's claim is revised down to the minimum of the remainder and the difference between her initial claim and her minimal right. Finally, the remainder is divided proportionally to the revised claims. This rule was originally introduced by Curiel, Maschler \& Tijs (1987) as the corresponding rule to a classical solution concept of cooperative games: the $\tau-$ value.

The next results follow:

\footnotetext{
${ }^{3}$ We do not include in the discussion the constrained equal losses rule because it trivially fails to satisfy securement, as there are problems for which agents with positive claims receive nothing.
} 
Proposition 1 The proportional rule satisfies bilateral consistency, self-duality and restricted path independence, and fails to satisfy securement.

\section{Proof.}

It is straightforward to show that the proportional rule is a bilaterally consistent and self-dual rule which satisfies path independence. In particular, it also satisfies restricted path independence. However, it does not satisfy securement. To see this, consider the bankruptcy problem $\bar{B}=$ $(\{1,2\}, 1,(1,3))$. Thus, $c_{1} \leq E$. However, $P_{1}(\bar{B})=\frac{1}{4}<\frac{1}{2}=\frac{c_{1}}{2}$.

Proposition 2 The constrained equal awards rule satisfies bilateral consistency, securement, and restricted path independence, and fails to satisfy selfduality.

\section{Proof.}

The constrained equal awards rule is a bilaterally consistent rule that satisfies path independence [c.f. Herrero \& Villar (2001, Th. 2)]. In particular, it also satisfies restricted path independence. Let us see that securement is also fulfilled. Let $i \in N$ be such that $c_{i} \leq E$. For the sake of contradiction, let us suppose that $A_{i}(N, E, c)=\lambda<\frac{1}{n} c_{i}<c_{i}$. Thus, $\lambda<\frac{E}{n}$. Now, for all $k \geq i$ we have $c_{k} \geq c_{i}$, which implies that $c_{k}>\lambda$ and therefore $A_{k}(N, E, c)=\lambda<\frac{E}{n}$. As a consequence,

$E=\sum_{k \in N} A_{k}(N, E, c)=\sum_{k=1}^{i-1} A_{k}(N, E, c)+\sum_{k=i}^{n} \lambda<\sum_{k=1}^{i-1} A_{k}(N, E, c)+(n-i+1) \frac{E}{n}$.

Thus, $\sum_{k=1}^{i-1} A_{k}(N, E, c)>\frac{i-1}{n} E$. Since $A_{k}(N, E, c) \geq 0$, for all $k \in N$, then there exists some $k_{0} \leq i-1$ such that $A_{k_{0}}(N, E, c)>\frac{E}{n}>\lambda=A_{n}(N, E, c)$, which is a contradiction, since $A$ is an order preserving rule [c.f. MorenoTernero \& Villar (2001, Proposition 2)].

Finally, it is well known that the constrained equal losses rule is the dual rule of $A$ [c.f. Moreno-Ternero \& Villar (2001, Corollary 1)] which implies that $A$ is not a self-dual rule.

Proposition 3 The adjusted proportional rule satisfies self-duality, securement and restricted path independence, and does not satisfy bilateral consistency.

\section{Proof.}

Curiel, Maschler \& Tijs (1987, Th. 4) show that $A P$ is a self-dual rule. It is straightforward to show that it also satisfies equal treatment of equals, 
a property that will be useful to show the fulfillment of restricted path independence.

Let $(N, E, c),\left(N, E^{\prime}, c\right) \in \mathbb{B}$ be such that $c_{1} \geq E^{\prime} \geq E$. If such is the case, then $m_{i}(N, E, c)=m_{i}\left(N, E^{\prime}, c\right)=0, t_{i}(N, E, c)=E$ and $t_{i}\left(N, E^{\prime}, c\right)=$ $E^{\prime}$, for all $i \in N$. Thus, $A P_{i}(N, E, c)=P_{i}(N, E,(E, \ldots, E))=\frac{E}{n}$ for all $i \in N$. Furthermore, $A P_{i}\left(N, E^{\prime}, c\right)=P_{i}\left(N, E^{\prime},\left(E^{\prime}, \ldots, E^{\prime}\right)\right)=\frac{E^{\prime}}{n}$ for all $i \in N$. Now, by equal treatment of equals, $A P_{i}\left(N, E, A P_{i}\left(N, E^{\prime}, c\right)\right)=$ $A P_{i}\left(N, E,\left(\frac{E^{\prime}}{n}, \ldots, \frac{E^{\prime}}{n}\right)\right)=\frac{E}{n}$ for all $i \in N$. Altogether shows that $A P$ satisfies restricted path independence.

To see that $A P$ satisfies securement, let $(N, E, c) \in \mathbb{B}$ and $i \in N$ be such that $c_{i} \leq E$. Let $j \in N$ be the first creditor whose minimal right is strictly positive, i.e., $j$ is such that $c_{j-1} \leq L<c_{j}$. In other words, $m_{k}(N, E, c)=0$ for all $k=1, \ldots, j-1$, and $m_{k}(N, E, c)=c_{k}-L$ for all $k=j, \ldots, n$. Thus, $E-\sum_{k \in N} m_{k}(N, E, c)=\sum_{k=1}^{j-1} c_{k}+(n-j) L$. It is then straightforward to show that $E-\sum_{k \in N} m_{k}(N, E, c)>c_{k}-m_{k}(N, E, c)$ for all $k \in N$. As a result,

$$
A P_{k}(N, E, c)=m_{k}(N, E, c)+P\left(N, E-\sum_{k \in N} m_{k}(N, E, c), c-m(N, E, c)\right),
$$

or equivalently,

$$
A P_{k}(N, E, c)=\left\{\begin{array}{ll}
\lambda \cdot c_{k} & \text { for all } k=1, \ldots, j-1 \\
c_{k}+(\lambda-1) L & \text { for all } k=j, \ldots, n
\end{array},\right.
$$

where

$$
\lambda=\frac{E-\sum_{k \in N} m_{k}(N, E, c)}{C-\sum_{k \in N} m_{k}(N, E, c)} .
$$

It is straightforward to show that $\lambda \geq \frac{1}{n}$. Now, if $i \leq j-1$ then $A P_{i}(N, E, c)=$ $\lambda \cdot c_{i} \geq \frac{1}{n} \cdot c_{i}$, as desired. If, on the other hand, $i \geq j$ then

$$
\begin{aligned}
A P_{i}(N, E, c) & =c_{i}+(\lambda-1) L=\frac{c_{i}}{n}+\frac{n-1}{n} c_{i}+(\lambda-1) L \\
& \geq \frac{c_{i}}{n}+\frac{n-1}{n} L+(\lambda-1) L \geq \frac{c_{i}}{n}+\left(\frac{n-1}{n}+\frac{1}{n}-1\right) L \geq \frac{c_{i}}{n},
\end{aligned}
$$

which shows that $A P$ satisfies securement.

Finally, note that $A P$ is a rule that coincides with the Talmud rule in the two-agent case. Since both are different rules, it follows from the "Elevator lemma" that it cannot be bilaterally consistent.

These Propositions show that there are rules that satisfy all the properties of the characterization result except securement (the proportional rule), 
rules that satisfy all the properties except self-duality (the constrained equal awards rule), and rules that satisfy all the properties except bilateral consistency (the adjusted proportional rule). To complete the analysis of the tightness of the characterization result we have to show that there are also rules that satisfy all the properties except restricted path independence. The following example illustrates this case and closes de discussion of the logical independence of the properties.

Example 1 Assume that we restrict our attention to the subset of two-agent bankruptcy problems. Without loss of generality assume $N=\{1,2\}$ and $c_{1} \leq c_{2}$. Consider the following rule:

$$
R(N, E, c)= \begin{cases}(0, E) & \text { if } E \leq \frac{c_{1}}{2} \\ \left(E-\frac{c_{1}}{2}, \frac{c_{1}}{2}\right) & \text { if } \frac{c_{1}}{2} \leq E \leq c_{1} \\ \left(\frac{c_{1}}{2}, E-\frac{c_{1}}{2}\right) & \text { if } c_{1} \leq E \leq c_{2} \\ \left(E-c_{2}+\frac{c_{1}}{2}, c_{2}-\frac{c_{1}}{2}\right) & \text { if } c_{2} \leq E \leq c_{2}+\frac{c_{1}}{2} \\ \left(c_{1}, E-c_{1}\right) & \text { if } c_{2}+\frac{c_{1}}{2} \leq E\end{cases}
$$

$R$ satisfies (vacuously) bilateral consistency. ${ }^{4}$ It is straightforward to show that $R$ is a self dual rule that satisfies securement. However, $R$ does not satisfy restricted path independence. Consider the vector of claims $c=(4,5)$, and the estates $E=1$ and $E^{\prime}=3$. Observe that $R(N, E, c)=(0,1)$ and $R\left(N, E^{\prime}, c\right)=(1,2)$. Thus, $R\left(N, E, R\left(N, E^{\prime}, c\right)\right)=R(N, 1,(1,2))=\left(\frac{1}{2}, \frac{1}{2}\right)$. As a result, $R(N, E, c) \neq R\left(N, E, R\left(N, E^{\prime}, c\right)\right)$.

Finally, we compare the characterization result provided here with other characterization results that appear in the literature. More precisely, let us refer to the work of Dagan (1996), who shows that the Contested Garment rule is the only two-person rule that satisfies self-duality and composition from minimal rights. ${ }^{5}$ From this result it follows that the Talmud rule is the only rule on $\mathbb{B}$ satisfying bilateral consistency, self-duality and composition from minimal rights. ${ }^{6}$ Proposition 2 shows that rule $A$ is a bilaterally consistent rule that satisfies securement. Yet, it does not satisfy composition from minimal rights [c.f. Moreno-Ternero \& Villar (2001, Proposition 3)].

\footnotetext{
${ }^{4}$ This line of argument has already been used by Moulin (2000).

${ }^{5}$ This property says that for every $(N, E, c) \in \mathbb{B}, F(N, E, c)=m(N, E, c)+F(N, E-$ $\left.\sum m_{i}(N, E, c), c-m(N, E, c)\right)$.

${ }^{6} \mathrm{He}$ also shows that the Contested Garment rule is the only two-person rule that satisfies self-duality and independence of claims truncation (resp. equal treatment of equals, independence of claims truncation and composition from minimal rights), where independence of claims truncation means that $F(N, E, c)=F(t(N, E, c))$, for all $(N, E, c) \in \mathbb{B}$. Applying bilateral consistency one gets the extension to the Talmud rule.
} 
Moreover, if we drop bilateral consistency, there are self-dual rules that satisfy both securement and composition from minimal rights which are not the Talmud rule (Proposition 3). The proportional rule is self-dual and bilaterally consistent and satisfies neither securement nor composition from minimal rights. Therefore the property of securement is logically independent of that of composition from minimal rights: it neither implies nor prevents the other property to be satisfied by a rule. As a consequence, the characterization result in Theorem 1 is genuinely different from that derived from the consistent extension of Dagan's results.

\section{References}

[1] Aumann, R.J. \& Maschler, M. (1985), Game theoretic analysis of a bankruptcy problem from the Talmud, Journal of Economic Theory, $36: 195-213$.

[2] Curiel, I.J., Maschler, M. \& Tijs, S.H. (1987), Bankruptcy games, Zeitschrift für Operations Research, 31 : A143-A159.

[3] Dagan, N. (1996), New characterizations of old bankruptcy rules, Social Choice and Welfare, $13: 51-59$.

[4] Herrero, C. \& Villar, A. (2001), The three musketeers: four classical solutions to bankruptcy problems, Mathematical Social Sciences, 42 : 307-328.

[5] Moreno-Ternero \& Villar, A. (2001), The TAL-family of rules for bankruptcy problems, IVIE Working Paper WP-AD 2001-33.

[6] Moulin, H. (1987), Equal or proportional division of a surplus, and other methods. International Journal of Game Theory, 16 (3) : 161-186.

[7] Moulin, H. (2000), Priority Rules and Other Asymmetric Rationing Methods, Econometrica, 68 : 643-684.

[8] Moulin, H. (2002), Axiomatic cost and surplus-sharing, In: Arrow, K., Sen, A., Suzumura, K., (Eds.), The Handbook of Social Choice and Welfare, Vol. 1. North-Holland. pp. 289-357.

[9] O'Neill, B. (1982), A problem of rights arbitration from the Talmud, Mathematical Social Sciences, 2 : 345-371. 
[10] Thomson, W. (1996), Consistent allocation rules, Mimeo, University of Rochester.

[11] Thomson, W. (2002), Axiomatic and game-theoretic analysis of bankruptcy and taxation problems: a survey, Mimeo, University of Rochester.

[12] Young, P. (1994), Equity, Princeton University Press, Princeton. 